\title{
Anti-tumor effects of vitamin D in glioblastoma: mechanism and therapeutic implications
}

\author{
Carmen Sze-Ching Lo ${ }^{1}$, Karrie Mei-Yee Kiang ${ }^{1}$ and Gilberto Ka-Kit Leung $\mathbb{D}^{1}{ }^{\text {布 }}$ \\ (C) The Author(s), under exclusive licence to United States and Canadian Academy of Pathology 2021
}

\begin{abstract}
Glioma is the most prevalent primary brain tumor in adults among which glioblastoma is the most malignant and lethal subtype. Its common resistance to conventional chemotherapeutics calls for the development of alternative or concomitant treatment. Taking advantage of its endocrine function as a neurosteroid, vitamin D has become a target of interest to be used in conjunction with different chemotherapies. In this article, we review the mechanisms through which vitamin D and its analogs induce anti-tumor activity in glioblastoma, and the practical issues relevant to their potential application based on in vitro and in vivo studies. Vitamin D has largely been reported to promote cell cycle arrest and induce cell death to suppress tumor growth in glioblastoma. Glioblastoma cells treated with vitamin D have also shown reduced migratory and invasive phenotypes, and reduced stemness. It is worth noting that vitamin D analogs are able to produce similar inhibitory actions without causing adverse effects such as hypercalcemia in vivo. Upregulation of vitamin D receptors by vitamin D and its analogs may also play a role in enhancing its antitumor activity. Based on current findings and taking into consideration its potential cancer-protective effects, the clinical application of vitamin D in glioblastoma treatment and prevention will be discussed. With some study findings subject to controversy, further investigation is warranted to elucidate the mechanism of action of vitamin $D$ and to evaluate relevant issues regarding its treatment efficacy and potential clinical application.
\end{abstract}

Laboratory Investigation (2022) 102:118-125; https://doi.org/10.1038/s41374-021-00673-8

\section{INTRODUCTION}

Gliomas, the most prevalent intracranial neoplasia in adults, account for around $50 \%$ of brain tumors $^{1-3}$. Glioblastoma, previously also known as glioblastoma multiforme (GBM), is the most malignant and life-threatening form. Classified as grade IV glioma by the World Health Organization (WHO), glioblastoma is associated with a median patient survival of 14-16 months, and a five-year survival rate of $9.8 \% \%^{4}$. While the new WHO CNS5 classification of gliomas has integrated histological appearances with molecular features and genetic alterations, the most aggressive subtype of glioma being an IDH-wildtype glioblastoma with the presence of at least one of the genetic parameters (TERT promoter mutation, EGFR gene amplification and combined chromosome + chr7/-chr10) in adults ${ }^{5}$. Apart from these genetic markers, other genetic risk factors including the expression of certain vitamin D receptors (VDR) genotypes were reported to be associated with clinical outcome of glioma patients ${ }^{6}$. Glioblastoma is also characterized by high invasiveness and the capability to diffusely invade into the normal cerebral parenchyma. Such an aggressive phenotype poses a hindrance to total en bloc resection and eventually a high risk of tumor relapse ${ }^{7,8}$.

Temozolomide (8-carbamoyl-3-methylidazo(5,1-d)-1,2,3,5-terrazin-4(3H)-one; TMZ) is a well-tolerated oral alkylating agent which has the ability to cross the blood-brain barrier ${ }^{9}$, making TMZbased chemoradiotherapy the current gold standard for the treatment of newly diagnosed glioblastoma ${ }^{10,11}$. However, treatment response remains suboptimal and is limited by its relatively short half-life $(1.8 \mathrm{~h})$. The expression of the DNA repairing enzyme methylguanine-DNA methyltransferase (MGMT) may compromise the cytotoxic effect of $T M Z$, leading to a high recurrence rate following TMZ treatment ${ }^{12}$. Strategies to complement response and overcome $T M Z$ resistance are therefore crucial and remain a cornerstone to improving patient prognosis ${ }^{13,14}$.

Current studies have suggested different strategies to enhance the chemosensitivity of TMZ. In addition to the use of synthetic compounds, many have studied the potential concomitant use of TMZ with dietary supplements ${ }^{8}$. One of such molecules is vitamin D. Exposure of the skin to sunlight leads to the formation of vitamin D3 (also referred to as cholecalciferol), which then undergoes bioactivation by double hydroxylation in the liver and kidney to form its active metabolite, calcitriol, or 1,25dihydroxyvitamin $D(1,25(\mathrm{OH}) 2 \mathrm{D} 3)^{15,16}$. Vitamin $\mathrm{D}$ has recently emerged as a neurosteroid hormone in the brain and has been shown to act as a regulator of a variety of brain functions, such as neuroprotection, anti-epileptic and anti-calcification effects, neuro-immunomodulation, and interplay with neurotransmitters and hormones. In particular, calcitriol regulates many cellular physiological processes, including cellular proliferation, differentiation, and apoptosis ${ }^{16}$. Calcitriol has shown anti-tumor effects in cancers of the pancreas, liver, breast, prostate, skin, brain, and myeloid leukemia ${ }^{17-20}$. However, the supraphysiological dosages of calcitriol required to produce the anti-neoplastic activity may cause side effects, including hypercalcemia, and may complicate cancer treatment ${ }^{17,21}$. In light of concerns regarding toxicity and

${ }^{1}$ Department of Surgery, Li Ka Shing Faculty of Medicine, The University of Hong Kong, Queen Mary Hospital, Hong Kong, Hong Kong. ${ }^{\circledR}$ email: gilberto@hku.hk 


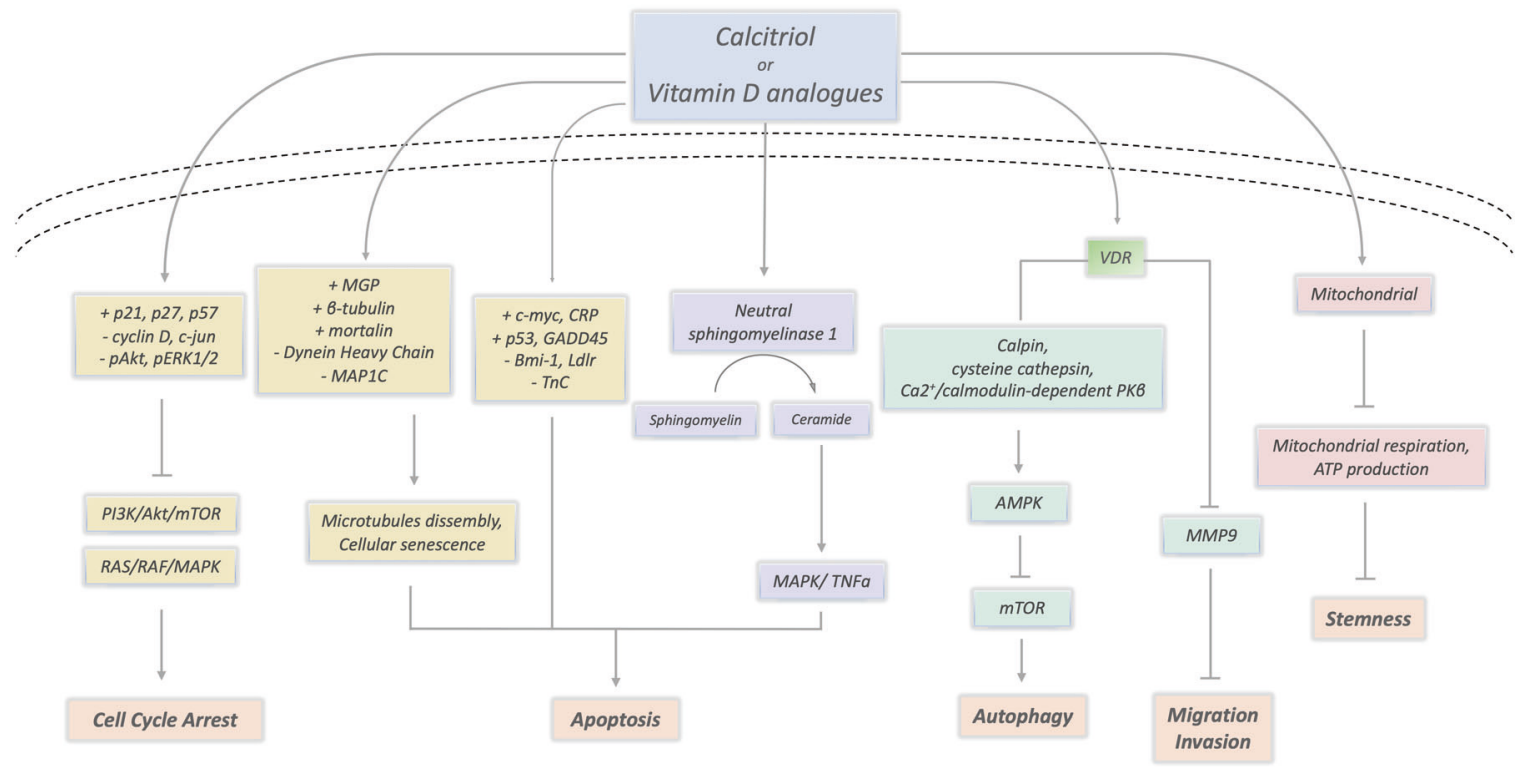

Fig. 1 Vitamin D-induced anti-tumoral actions in cell proliferation, apoptosis, migration, invasion, and stemness. Calcitriol or vitamin D analogs inhibit cell cycle transition, directly through upregulating cyclin-dependent kinase inhibitors, or indirectly through regulating other growth factors. It also induces apoptosis via the activation of intrinsic apoptotic pathways, tumor-suppressor pathways, and the ceramide pathway, and induces VDR-mediated autophagy. In addition, calcitriol acts on VDR to suppress MMP activity to inhibit tumor migration and invasion and interferes with mitochondrial respiration to repress stemness. (+ Upregulation - Downregulation).

calcium homeostasis, the application of vitamin D analogs with milder systemic side effects have also been proposed as an alternative. In addition to empirical evidence substantiating its anti-tumor effects, empirical and epidemiological studies have proposed that vitamin D may play a chemopreventive role in prostate, breast, colorectal cancers and gliomas, and put forth a possibility of application in cancer prevention ${ }^{18,22}$. In this review article, we discuss how vitamin $D$ exerts its function in glioblastoma cells, with a focus on the molecular mechanisms underlying its anti-tumor effects, and most importantly, the therapeutic implications.

\section{GENOMIC AND NON-GENOMIC ACTIONS OF VITAMIN D}

Classically, vitamin D and its analogs act through the genomic pathway which involves vitamin D receptors (VDRs). VDRs are present in more than 30 different tissues throughout the body, including the intestine, bone, brain, stomach, heart, pancreas, skin, activated $\mathrm{T}$ and $\mathrm{B}$ lymphocytes, and gonads ${ }^{23}$. Upon binding of the hormonally active vitamin $\mathrm{D}\left(1,25(\mathrm{OH})_{2} \mathrm{D} 3\right)$ to VDR, which is a ligand-activated transcription factor in the inner nuclear membrane microdomains, retinoid-X receptor (RXR) or in some cases the retinoid-A receptor (RAR) facilitates its binding to the vitamin $D$ response element (VDRE) in target gene promoters ${ }^{24-26}$. The assembly of VDR/RXR heterodimers can activate transcription in different genes containing functional VDREs, such as 25(OH)D3 24hydroxylase ${ }^{27}$, and those involved in cell proliferation, including p21 $28, \mathrm{GADD}^{28} 5^{29}$, and TNFa ${ }^{30}$.

Apart from the classical VD-VDR response, vitamin D can also activate receptor-independent non-genomic pathways to regulate cytoplasmic signaling and mediate cellular functions. These signaling pathways include protein kinase $C^{31}$, Ras and mitogenactivated protein kinase $(\mathrm{MAPK})^{31,32}$, and the ceramide pathway ${ }^{33,34}$. Such non-genomic actions lead to rapid intracellular changes in calcium, as well as the activation or deactivation of proteins like $\mathrm{BCl}-2$ and $\mathrm{c}$-jun, thereby regulating cellular growth, differentiation, and apoptosis, independently or in cooperation with the classical genomic VDR pathway ${ }^{21}$.

\section{MECHANISMS OF VITAMIN D ANTI-TUMOR ACTIVITY}

A number of vitamin $D$ studies performed on in vitro and in vivo cancer models have proposed several mechanisms in explanation of the tumor-suppressive activity of vitamin D and its analogs in glioma. Figure 1 illustrates the anti-tumoral mechanisms of vitamin $D$ in glioblastoma, and Table 1 summarizes the antitumor mechanisms of calcitriol and its analogs.

\section{CELL CYCLE ARREST}

Cell cycle arrest is one of the most well-studied mechanisms accounting for the anti-tumor activity of vitamin D in gliomas. Several studies have demonstrated that calcitriol-induced arrest in the G0/G1 phase in various glioblastoma cell lines ${ }^{35-}$ 37. Treatments with its analogs have shown similar cytostatic effects. For example, EM1 and tacalcitol inhibited glioblastoma cell proliferation in T98G cell line ${ }^{35,38}$, and ML-344 induced cell cycle arrest in the murine GL261 and human U251 cell lines ${ }^{36}$. Such inhibitory effects on cell cycle progression were suggested to be induced by the simultaneous activation of cyclin-dependent kinase (CDK) inhibitors p21, p27 and p5 $57^{6,21,38-40}$, as well as the reduction in cyclin D1 and c-jun expressions, and the reduction in phosphorylation of pAkt (S473) and pERK1/2 (Thr202/Tyr204) ${ }^{6,34,38}$, thereby affecting downstream signal transduction to suppress glioblastoma cell proliferation $^{38}$. Known to play a crucial role in glioma tumorigenesis, the overexpression and/or mutation of epidermal growth factor receptor (EGFR) can activate downstream targets via multiple signaling pathways, including the phosphatidylinositol 3-kinase (PI3K)/Akt/rapamycin-sensitive mTORcomplex (mTOR) pathway, the RAS/RAF/MAPK pathway, together with their key phosphorylated kinases ${ }^{38,41,42}$. By delaying the phosphorylation of Akt at Ser473 (p-Akt-S473) and ERK1/2 at Thr202/Tyr204 (pERK1/2), and simultaneously decreasing the expression of nuclear transcription factor c-jun, a downstream substrate of MAPKs, calcitriol and its analogs may interfere with signal transduction and ultimately inhibit proliferation of glioma cells. 


$$
\dot{\mathrm{s}}:
$$

$\hat{m}$

in

$\infty$

in

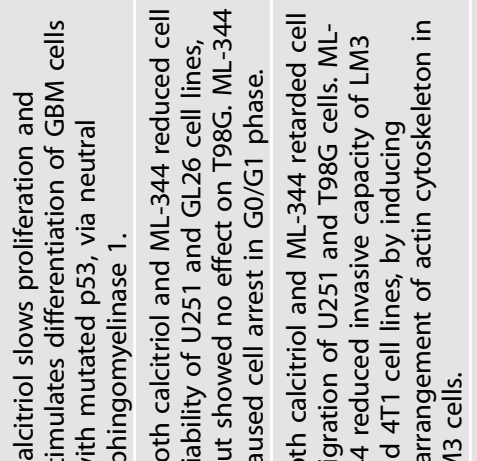

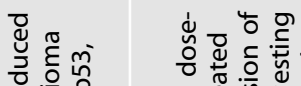

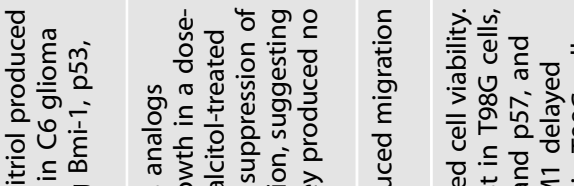

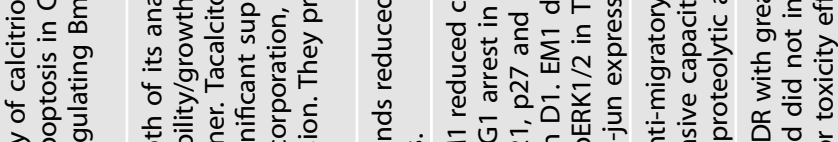

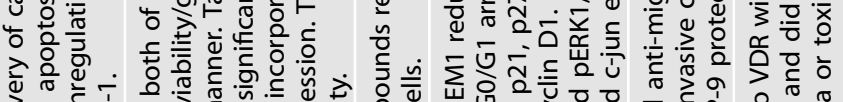

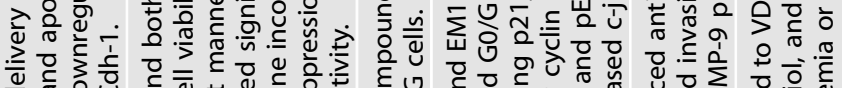

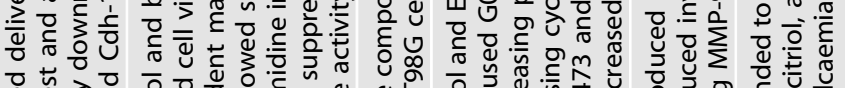

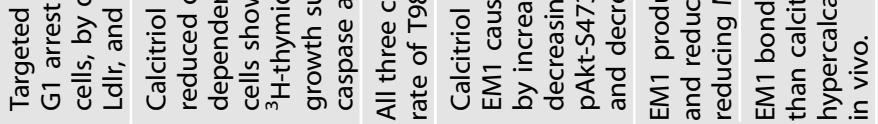

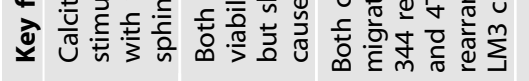

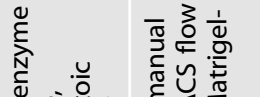

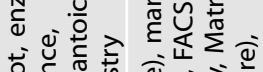

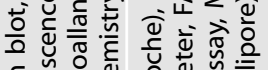

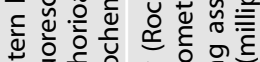

पे

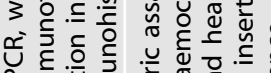

운.

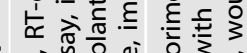

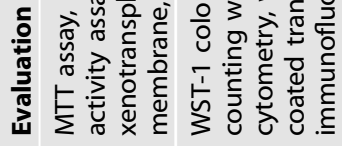

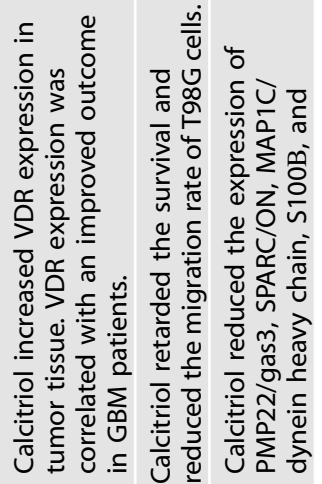
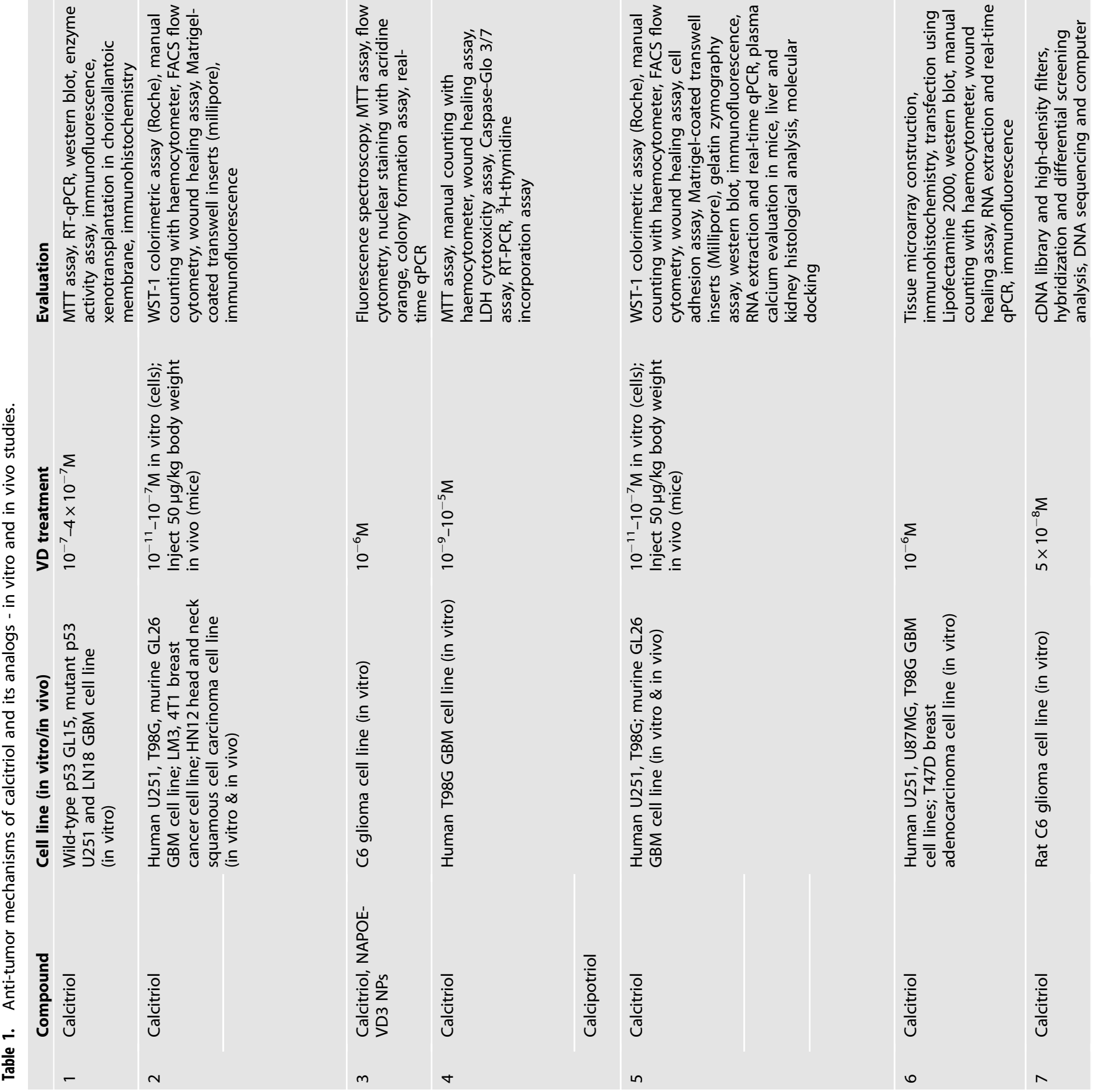
in $\quad$ \%

$\stackrel{\infty}{\rightarrow}$ i

$\stackrel{0}{\wedge}$

\%

ชิ

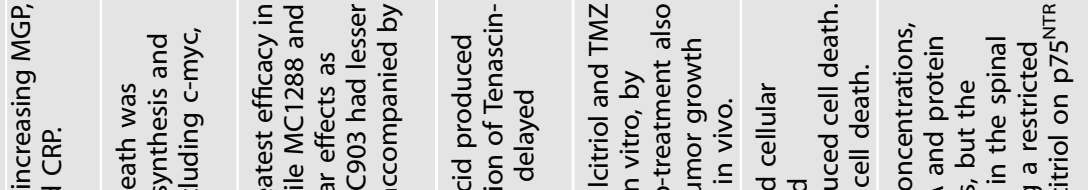

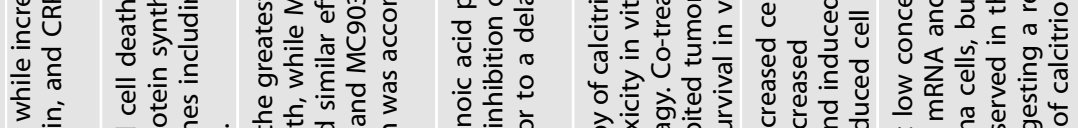

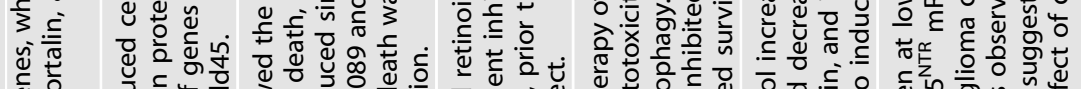

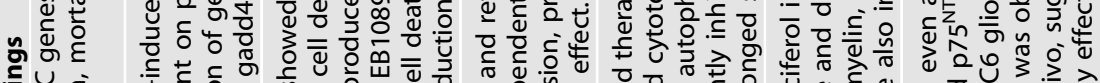

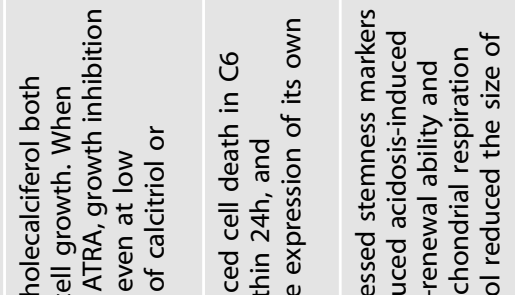

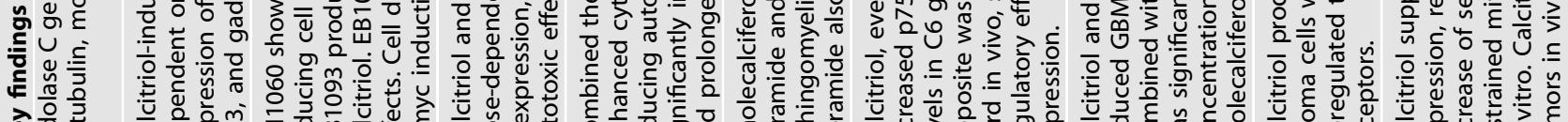

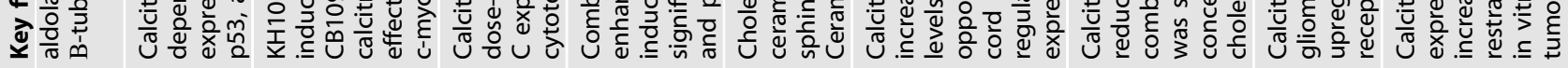
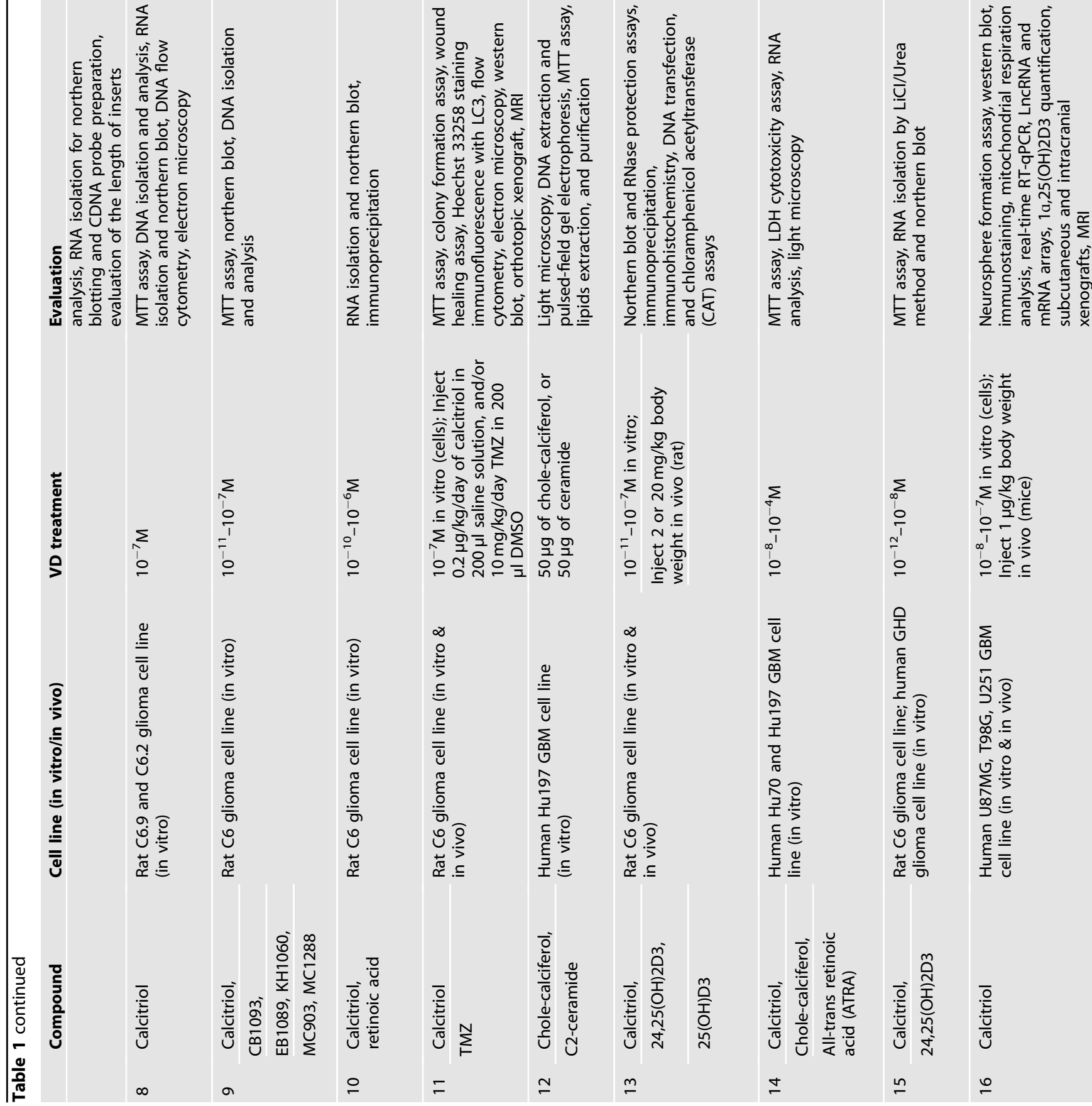


\section{AUTOPHAGY AND APOPTOSIS}

Cytotoxic autophagy has been suggested to be an important apoptotic mechanism of calcitriol in glioblastoma, where it demonstrates synergistic effects with $\mathrm{TMZ}^{43}$. Bak et al. observed in calcitriol-treated $\mathrm{C} 6$ cells the ultrastructure of autophagosomes and found an increase in size and number, along with an increase in conversion of microtubule-associated protein 1 light chain 3 puncta LC3-I to LC3-II, indicating the activation of autophagy ${ }^{43}$. Consistently, in breast cancer cells treated with calcitriol and its analog EB1089, Høyer-Hansen et al. found damaged mitochondria containing markers indicative of autolysosomes, including cathepsin D, heat-shock protein 60 , and cytochrome $c^{44}$. It has been suggested that VDR mediated the elevation of cytoplasmic $\mathrm{Ca}^{2+}$ concentration by altering calcium-regulating protein expression. Such an increase induces and activates calpain, cysteine cathepsins, and $\mathrm{Ca}^{2+}$ /calmodulin-dependent protein kinase- $\beta$ which subsequently activates AMP-activated kinase $(A M P K)^{44,45}$. AMPK acts as a potent inducer of autophagy via the inhibition of mammalian target of rapamycin (mTOR) complex 1, followed by the activation of several autophagy-associated proteins, leading to massive cell death ${ }^{45,46}$. This autophagy-induced anti-tumor effect has been proposed to be beclin-dependent, with the alteration of $\mathrm{BCl}-1 / \mathrm{Bcl}-2$ balance restoring autophagic activity to normal levels in tumor cells. Bcl-1 is a tumor suppressor and is negatively regulated by $\mathrm{Bcl}-2$, an anti-apoptotic protein overexpressed in many tumors ${ }^{44}$. Høyer-Hansen et al. demonstrated an increase in Bcl-1 activity which enhanced DNA fragmentation and chromatin condensation in breast cancer cells, and Guzey et al. demonstrated the downregulation of originally overexpressed $\mathrm{Bcl}-2$ in several prostate cancer cell lines, in response to calcitriol treatment ${ }^{44,47}$. This cell death process may be accelerated by an accompanying increase in autophagic stress, further raising autophagic activity above normal levels ${ }^{43}$. While the precise pathway by which enhanced autophagic activity promotes glioma cell death remains to be determined, these findings nonetheless support its key involvement in inducing apoptosis in glioma.

Another apoptotic pathway activated by calcitriol is the ceramide pathway. Cataldi et al. demonstrated in glioblastoma cell lines U251 and LN18 that upon calcitriol treatment, neutral sphingomyelinase 1 degraded sphingomyelin which increased intracellular ceramide level ${ }^{34}$. Cholecalciferol, a vitamin D metabolite, activated the sphingomyelin pathway and led to a prominent increase in ceramide levels in a similar manner in Hu197 cells, followed by massive cell death ${ }^{48}$. This was first shown by Okazaki et al. in leukemia cell line $\mathrm{HL}-60^{33,49}$, and it was proposed that the dysregulation of the balance between ceramide and other sphingolipids exerting opposing effects on MAPKs may activate an intrinsic cell death pathway ${ }^{48}$. Pirianov et al. further demonstrated in breast cancer cells that vitamin D analog CB1093 was able to potentiate TNFa-induced cytotoxicity, possibly by promoting $\mathrm{C}_{2}$-ceramide-induced DNA fragmentation and cytosolic phospholipase A2 (CPLA $)$ activation ${ }^{50}$. Despite the limited understanding of the interaction between VD, ceramide and its downstream signals, it is surmised that vitamin D activates sphingomyelinase to induce intracellular ceramide, that in turn promotes apoptosis through TNFa/MAPK pathways in glioblastoma cells.

VD exerts its effect via multiple modes of action, suggesting the involvement of various apoptotic players in addition to the autophagic and ceramide pathways. Calcitriol has been reported to alter the expression of several house-keeping genes involved in maintaining cell structure, motility and defense. Baudet et al. demonstrated in $\mathrm{C} 6$ rat glioma that calcitriol upregulated matrix Gla protein (MGP), $\beta$-tubulin and mortalin, causing microtubule disassembly, cellular senescence and cell death ${ }^{51}$. It simultaneously downregulated Dynein Heavy Chain/MAP1C, responsible for the microtubules-associated intracellular movements. Hence, calcitriol introduced perturbation in basic cellular functions that led to glioblastoma cell death. This is consistent with the observed reduction in osteonectin/SPARC expression, which primarily functions to enhance cell viability under stressful conditions.

On the other hand, calcitriol has been shown to regulate the expression of genes that participate in the course of apoptosis or programmed cell death. Calcitriol increased the expression of the proto-oncogene c-myc and its primary response gene cysteinerich protein (CRP), tumor-suppressor gene $\mathrm{p} 53$, and growth arrest and DNA damage-inducible gene GADD45, hence inducing apoptosis or programmed cell death following irreversible DNA damage ${ }^{52,53}$, while simultaneously downregulating protooncogene Bmi-1 and Ldlr responsible for the energy nourishment of glioma cells $s^{37,54}$. In addition, Alvarez-Dolado et al. demonstrated that calcitriol suppressed the expression of Tenascin-C (TnC), an extracellular matrix glycoprotein normally involved in glial cell development but substantially reactivated in glioma cells, triggering tumor growth, invasion, and angiogenesis. Its downregulation subsequently produced delayed cytotoxicity in C6 rat glioma cells $s^{55}$.

The upregulation of neurotrophin expression has also been linked to calcitriol-induced cell death. The overexpression of lowaffinity neurotrophin receptor $\mathrm{p} 75^{\mathrm{NTR}}$ following calcitriol treatment, in the absence of its ligand, can induce apoptosis directly, or indirectly via the activation of the ceramide pathway given its structural homology with the TNF receptor (TNFR) ${ }^{56-58}$. IL-6, an inflammatory cytokine with reported neurotrophic properties, was upregulated by calcitriol and may act to cause cell death by modifying the host-glioma immune interaction ${ }^{52}$. These are consistent with the observed increase, in response to calcitriol, in vascular endothelial growth factor (VEGF) expression, a hypoxiainducible neurotrophic factor upregulated in stressful conditions such as programmed cell death. The role of certain calcitriolinduced neurotrophins in glioma is, however, reported to be contradictory to its potential cell-protective effects and will be further discussed in this article.

\section{ANTI-MIGRATORY AND ANTI-INVASIVE EFFECTS}

Glioblastoma is known for its highly invasive nature, and is characterized by its extensive infiltration into the surrounding normal brain tissue ${ }^{59}$. Its invasion is often described as a multistep process, that involves first the adhesion of tumor cells to the extracellular matrix (ECM), the secretion of matrix metalloproteinases (MMPs) to degrade the local ECM, and subsequently the migration into the proteolytically modified $\mathrm{ECM}^{60}$. Calcitriol and its analogs have demonstrated anti-migratory effects and decreased invasive capacity in this regard. Salomón et al. demonstrated that calcitriol lowered T98G cell migration rate, and that the reduction was VDR-dependent ${ }^{6}$. Such suppression of migration by calcitriol through VDR may be attributed to the translocation of tumorsuppressor p27 to the nucleus ${ }^{6,61}$. Ferronato et al., on the other hand, demonstrated that calcitriol analog EM1 reduced MMP9 proteolytic activity in T98G cells, hence suppressing glioblastoma infiltration through the surrounding ECM into normal tissue ${ }^{38}$. Similarly, ML-344 has been shown to retard migration and reduce invasiveness in U251 and T98G glioma cell lines, as well as breast and head and neck cancer cell lines ${ }^{36}$.

\section{REPRESSION OF STEMNESS}

The acidic microenvironment in glioblastoma is associated with the maintenance of cancer stemness and facilitates tumor progression. CYP24A1 encoding for 25-hydroxyvitamin D3-24hydroxylase, which catalyzes the fast degradation of calcitriol, was overexpressed in stem cell-like glioma cells (SLCs) under acidosis and was accounted for their stemness and metabolism changes. $\mathrm{Hu}$ et al. revealed that calcitriol could repress stemness and the self-renewal ability of SLCs, by inhibiting mitochondrial respiration 
and ATP production ${ }^{62}$. It is suggested that calcitriol acts to inhibit stemness markers expression and attenuate the acidosis-induced increase of self-renewal ability and mitochondrial respiration of glioma stem cells (GSCs) in malignant glioma, in a similar manner.

\section{THE ROLE OF VDR IN GLIOMA}

VD has been shown to upregulate VDR expression in tumor tissues, which further enhances its anti-neoplastic effects. Salomón et al. showed that silencing of VDR significantly increased cellular survival of T98G. However, subsequent exposure to calcitriol augmented VDR mRNA and protein levels and suppressed glioma cell survival in U251, U87MG and T98G cell lines, suggesting antitumor signaling mediated by VDR ${ }^{6}$. Such an increase in VDR expression stimulated by calcitriol has also been demonstrated in C6 glioma cells where it was accompanied by apoptotic cell death, characterized by DNA fragmentation and elevated protooncogenes p53 and c-myc expression ${ }^{52,63}$. This is in agreement with that observed in other cervical, mammary and ovarian cancers $^{64-66}$. In addition, Davoust et al. demonstrated that stable transfection of VDR into a VD-resistant rat glioma clone was able to restore its sensitivity to calcitriol-induced cytotoxicity ${ }^{67}$, not only corroborating the role of VDR in producing VD-mediated antitumor activity in glioma, but also proposing a solution to overcoming resistance to vitamin D by inducing VDR expression in glioma.

\section{CONTRADICTORY PROTECTIVE EFFECTS}

On the other hand, calcitriol has been reported to exert contradictory protective effects on glioma cells, mainly through the effect of neurotrophins. Calcitriol stimulates the production of numerous trophic factors including nerve growth factors (NGFs) ${ }^{68}$, neurotrophin-3 (NT-3) ${ }^{69}$, and glial cell line-derived neurotrophic factor $(\mathrm{GDNF})^{70}$, all of which enhance survival of glioma cells. NGFs have previously been observed to increase the proliferation of human glioma cell lines U87, U251 and U373 ${ }^{71}$, possibly via a9B1 integrin $^{72}$. NT-3 has been shown to be protective of cells with the potential to initiate glial tumor growth ${ }^{73}$, and of glioma cells in a hypoxic environment ${ }^{74}$. Blockage of GDNF- and GDNF receptor-a1 expression has been reported to inhibit $\mathrm{C} 6$ rat glioma cell proliferation ${ }^{75}$, suggesting the potential promoter role of GDNF in glioma progression. Conversely, calcitriol has also been shown to downregulate NT-4 and to have no effect on the expression of $\mathrm{BDNF}^{69}$. Accumulating evidence suggested the potential role of neurotrophins in glioma progression, but such oncogenic functions remain largely unknown. Further investigation is needed to evaluate the net consequences on cell survival contributed by these neurotrophins.

\section{THERAPEUTIC IMPLICATIONS}

Empirical evidence shows that calcitriol and cholecalciferol exert therapeutic effects on its own at concentrations of $10^{-8}$ to $10^{-4} \mathrm{M}$, which carry a potential risk of causing hypercalcemia ${ }^{21}$. Therefore, efforts have been made to identify safer alternatives. Several vitamin $D$ synthetic analogs have been examined, including tacalcitol, calcipotriol, ML-344, EM1, CB1093, EB1089, KH1060, MC903 and MC1288, all of which have proved to be able to produce similar therapeutic effects without giving rise to severe hypercalcemic side effects.

When combined with other known chemotherapeutic agents, calcitriol and cholecalciferol have demonstrated synergy in terms of therapeutic efficacy. Examples include the combination of calcitriol with temozolomide, and calcitriol or cholecalciferol with retinoic acid ${ }^{43,55,76}$. Concomitant treatment with vitamin $D$ or vitamin $D$ on its own may serve as a new therapeutic paradigm to overcoming chemoresistance in glioblastoma. Several phase I/II clinical trials on the combination therapy of calcitriol and other chemotherapeutic agents on glioma and other solid tumors have been taking place. A phase I/II trial is in progress to assess the efficacy and toxicity of long-term high-dose vitamin D3 with concurrent chemoradiotherapy/adjuvant chemotherapy containing TMZ in patients with newly diagnosed glioblastoma, evaluating doses as high as $40001 \mathrm{U}^{77}$. Another phase I trial also underway aims to determine the effectiveness and maximum tolerated doses of subcutaneous and/or oral calcitriol combined with intravenous carboplatin in treating advanced solid tumors, including brain tumors ${ }^{78}$. While these ongoing clinical trials are still awaiting results, they shall provide valuable information regarding the efficacy, toxicity, and other practical issues to evaluate the potential clinical application of VD.

Based on the role of VDR as a regulator of autophagy, recent studies have also suggested a potential cancer-preventive effect of VD. Tavera-Mendoza et al. observed that in addition to inducing autophagy in breast cancer cells, dietary vitamin D supplementation in mice was able to increase basal autophagy levels even in the normal mammary gland, highlighting its protective effect against cancer ${ }^{79}$. Indeed, epidemiological studies have reported an inverse relationship between pre-diagnostic $25(\mathrm{OH}) \mathrm{D}$ levels and glioblastoma risk ${ }^{22}$. However, empirical glioma studies are required to elucidate this preventive effect in glioblastoma. VDR gene polymorphisms Fok-I (rs2228570) and Taq-I (rs731236) have also been reported as a genetic risk factor in various cancer types. Toptas et al. demonstrated a positive correlation between a VDR Fok-I ff genotype and the risk of meningioma ${ }^{80}$. Despite there being no significant difference observed in glioma patients, a larger scale genome-wide association study between VDR polymorphism and glioma susceptibility is needed. The functional and prognostic significance of VDR is becoming more prominent as VDR expression was also associated with KRAS mutation in several cancers ${ }^{81}$. Together, these findings point to the potential dual role of vitamin $D$ as both a therapeutic and prophylactic agent against glioblastoma and support the clinical application of vitamin D or VDR as a novel biomarker in this regard.

To date, no unifying theories regarding the anti-tumor activity of vitamin $D$ is available. Nevertheless, the mechanism by which vitamin D produces anti-tumor activity in glioma is likely to entail both genomic and non-genomic pathways and involve a vast number of downstream mediators and effectors. Robust evidence supports that vitamin D and its analogs may act to produce cell cycle arrest, apoptosis, anti-migratory and anti-invasive effects and repression of stemness, while upregulating VDR to further enhance anti-tumor response. On the other hand, some, though limited, evidence suggests a protective role of vitamin D on cancer patient survival. More investigation into the potential dual role and subsequent net effect of vitamin D on glioma progression is warranted. Regardless, current evidence presents a favorable and promising outlook on the therapeutic application of vitamin D and its analogs as a supplement to standard glioblastoma therapy, along with a potential application in glioblastoma prevention, offering insight into new means of overcoming chemoresistance and improving glioma patient survival.

\section{DATA AVAILABILITY}

Data sharing is not applicable to this article as no datasets were generated or analyzed during the current study.

\section{REFERENCES}

1. Demuth, T. \& Berens, M. Molecular mechanisms of glioma cell migration and invasion. J. Neurooncol. 70, 217-228 (2004).

2. Sathornsumetee, S. \& Rich, J. New treatment strategies for malignant gliomas. Expert Rev. Anticancer Ther. 6, 1087-1104 (2006). 
3. Mahaley, J., Mettlin, C., Natarajan, N., Laws, J. \& Peace, B. National survey of patterns of care for brain-tumor patients. J. Neurosurg. 71, 826-836 (1989).

4. Stupp, R. et al. Effects of radiotherapy with concomitant and adjuvant temozolomide versus radiotherapy alone on survival in glioblastoma in a randomised phase III study: 5-year analysis of the EORTC-NCIC trial. Lancet Oncol. 10, 459-466 (2009).

5. Louis, D. et al. The 2021 WHO Classification of Tumors of the Central Nervous System: a summary. Neuro. Oncol. 23, 1231-1251 (2021).

6. Salomon, D. et al. Vitamin D receptor expression is associated with improved overall survival in human glioblastoma multiforme. J. Neurooncol. 118, 49-60 (2014).

7. Elmaci, I. \& Altinoz, M. A metabolic inhibitory cocktail for grave cancers: metformin, pioglitazone and lithium combination in treatment of pancreatic cancer and glioblastoma multiforme. Biochem. Genet. 54, 573-618 (2016).

8. Elmaci, I., Ozpinar, A., Ozpinar, A., Perez, J. \& Altinoz, M. From epidemiology and neurometabolism to treatment: Vitamin $D$ in pathogenesis of glioblastoma Multiforme (GBM) and a proposal for Vitamin D + all-trans retinoic acid + Temozolomide combination in treatment of GBM. Metab. Brain. Dis. 34, 687-704 (2019).

9. Newlands, E. et al. Phase I trial of temozolomide (CCRG 81045: M\&B 39831: NSC 362856). Br J. Cancer 65, 287-291 (1992).

10. Nachbichler, S., Schupp, G., Ballhausen, H., Niyazi, M. \& Belka, C. Temozolomide during radiotherapy of glioblastoma multiforme: daily administration improves survival. Strahlenther Onkol. 193, 890-896 (2017).

11. Dehdashti, A., Hegi, M., Regli, L., Pica, A. \& Stupp, R. New trends in the medical management of glioblastoma multiforme: the role of temozolomide chemotherapy. Neurosurg. Focus 20, E6 (2006).

12. Zhang, J., Stevens, M. \& Bradshaw, T. Temozolomide: mechanisms of action, repair and resistance. Curr. Mol. Pharmacol. 5, 102-114 (2012).

13. Begemann, M. et al. Growth inhibition induced by Ro 31-8220 and calphostin $C$ in human glioblastoma cell lines is associated with apoptosis and inhibition of CDC2 kinase. Anticancer Res. 18, 3139-3152 (1998).

14. Gagliano, N. et al. Ukrain modulates glial fibrillary acidic protein, but not connexin 43 expression, and induces apoptosis in human cultured glioblastoma cells. Anticancer Drugs 18, 669-676 (2007).

15. Garcion, E., Wion-Barbot, N., Montero-Menei, C., Berger, F. \& Wion, D. New clues about vitamin D functions in the nervous system. Trends Endocrinol. Metab. 13, 100-105 (2002).

16. Kalueff, A. \& Tuohimaa, P. Neurosteroid hormone vitamin D and its utility in clinical nutrition. Curr. Opin. Clin. Nutr. Metab. Care 10, 12-19 (2007).

17. Beer, T. et al. Double-blinded randomized study of high-dose calcitriol plus docetaxel compared with placebo plus docetaxel in androgen-independent prostate cancer: a report from the ASCENT investigators. J. Clin. Oncol. 25, 669-674 (2007).

18. Guyton, K., Kensler, T. \& Posner, G. Vitamin D and vitamin D analogs as cancer chemopreventive agents. Nutr. Rev. 61, 227-238 (2003).

19. Holick, M. Vitamin D and sunlight: strategies for cancer prevention and other health benefits. Clin. J. Am. Soc. Nephrol. 3, 1548-1554 (2008).

20. Johnson, C., Hershberger, P., Bernardi, R., McGuire, T. \& Trump, D. Vitamin D receptor: a potential target for intervention. Urology 60, 123-130 (2002). discussion 130-121.

21. Beer, T. \& Myrthue, A. Calcitriol in cancer treatment: from the lab to the clinic. Mol. Cancer Ther. 3, 373-381 (2004).

22. Zigmont, V. et al. Association between prediagnostic serum 25 -hydroxyvitamin $D$ concentration and glioma. Nutr. Cancer 67, 1120-1130 (2015).

23. Holick, M. Vitamin D: a millenium perspective. J. Cell Biochem. 88, 296-307 (2003).

24. Bartoccini, E. et al. Nuclear lipid microdomains regulate nuclear vitamin D3 uptake and influence embryonic hippocampal cell differentiation. Mol. Biol. Cell 22, 3022-3031 (2011)

25. Kliewer, S., Umesono, K., Mangelsdorf, D. \& Evans, R. Retinoid X receptor interacts with nuclear receptors in retinoic acid, thyroid hormone and vitamin D3 signalling. Nature 355, 446-449 (1992).

26. Pike, J. \& Meyer, M. The vitamin D receptor: new paradigms for the regulation of gene expression by 1,25-dihydroxyvitamin D(3). Endocrinol. Metab. Clin. North Am. 39, 255-269 (2010). table of contents.

27. Zierold, C., Darwish, H. \& Deluca, H. Identification of a vitamin D-response element in the rat calcidiol (25-hydroxyvitamin D3) 24-hydroxylase gene. Proc. Natl. Acad. Sci. USA 91, 900-902 (1994).

28. Liu, M., Lee, M., Cohen, M., Bommakanti, M. \& Freedman, L. Transcriptional activation of the Cdk inhibitor p21 by vitamin D3 leads to the induced differentiation of the myelomonocytic cell line U937. Genes Dev. 10, 142-153 (1996).

29. Jiang, F., Li, P., Fornace, A., Nicosia, S. \& Bai, W. G2/M arrest by 1,25-dihydroxyvitamin D3 in ovarian cancer cells mediated through the induction of GADD45 via an exonic enhancer. J. Biol. Chem. 278, 48030-48040 (2003).
30. Hakim, I. \& Bar-Shavit, Z. Modulation of TNF-alpha expression in bone marrow macrophages: involvement of vitamin D response element. J. Cell Biochem. 88, 986-998 (2003).

31. Beno, D., Brady, L., Bissonnette, M. \& Davis, B. Protein kinase C and mitogenactivated protein kinase are required for 1,25-dihydroxyvitamin D3-stimulated Egr induction. J. Biol. Chem. 270, 3642-3647 (1995).

32. Park, W. et al. Induction of apoptosis by vitamin D3 analogue EB1089 in NCl-H929 myeloma cells via activation of caspase 3 and p38 MAP kinase. Br. J. Haematol. 109, 576-583 (2000).

33. Okazaki, T., Bielawska, A., Bell, R. \& Hannun, Y. Role of ceramide as a lipid mediator of 1 alpha,25-dihydroxyvitamin D3-induced HL-60 cell differentiation. J. Biol. Chem. 265, 15823-15831 (1990).

34. Cataldi S., et al. Effect of 1alpha,25(OH)2 vitamin D3 in mutant P53 glioblastoma cells: involvement of neutral sphingomyelinase1. Cancers 12, 3163 (2020).

35. Emanuelsson, I., Wikvall, K., Friman, T. \& Norlin, M. Vitamin D analogues tacalcitol and calcipotriol inhibit proliferation and migration of T98G human glioblastoma cells. Basic Clin. Pharmacol. Toxicol. 123, 130-136 (2018).

36. Ferronato, M. et al. Synthesis of a novel analog of calcitriol and its biological evaluation as antitumor agent. J. Steroid. Biochem. Mol. Biol. 185, 118-136 (2019).

37. Maleklou, N., Allameh, A. \& Kazemi, B. Preparation, characterization and in vitrotargeted delivery of novel Apolipoprotein E-based nanoparticles to C6 glioma with controlled size and loading efficiency. J. Drug Target 24, 348-358 (2016).

38. Ferronato, M. et al. Antitumoral effects of the alkynylphosphonate analogue of calcitriol EM1 on glioblastoma multiforme cells. J. Steroid Biochem. Mol. Biol. 178 , 22-35 (2018).

39. Vuolo, L., Di Somma, C., Faggiano, A. \& Colao, A. Vitamin D and cancer. Front. Endocrinol. 3, 58 (2012).

40. Wang, Q. M., Jones, J. B. \& Studzinski, G. P. Cyclin-dependent kinase inhibitor p27 as a mediator of the G1-S phase block induced by 1,25-dihydroxyvitamin D3 in HL60 cells. Cancer Res. 56, 264-267 (1996).

41. Gao, Q., Lei, T. \& Ye, F. Therapeutic targeting of EGFR-activated metabolic pathways in glioblastoma. Expert Opin. Investig. Drugs 22, 1023-1040 (2013).

42. Li, X. et al. PI3K/Akt/mTOR signaling pathway and targeted therapy for glioblastoma. Oncotarget 7, 33440-33450 (2016).

43. Bak, D. et al. Autophagy enhancement contributes to the synergistic effect of vitamin $\mathrm{D}$ in temozolomide-based glioblastoma chemotherapy. Exp. Ther. Med. 11, 2153-2162 (2016).

44. Hoyer-Hansen, M., Bastholm, L., Mathiasen, I., Elling, F. \& Jaattela, M. Vitamin D analog EB1089 triggers dramatic lysosomal changes and Beclin 1-mediated autophagic cell death. Cell Death Differ. 12, 1297-1309 (2005).

45. Picotto, G., Liaudat, A., Bohl, L., Tolosa \& de Talamoni, N. Molecular aspects of vitamin D anticancer activity. Cancer Invest. 30, 604-614 (2012).

46. Hoyer-Hansen, M., Nordbrandt, S. \& Jaattela, M. Autophagy as a basis for the health-promoting effects of vitamin D. Trends Mol. Med. 16, 295-302 (2010).

47. Guzey, M., Kitada, S. \& Reed, J. Apoptosis induction by 1alpha,25-dihydroxyvitamin D3 in prostate cancer. Mol. Cancer Ther. 1, 667-677 (2002).

48. Magrassi, L. et al. Vitamin D metabolites activate the sphingomyelin pathway and induce death of glioblastoma cells. Acta Neurochir. 140, 707-713 (1998). discussion 713-704.

49. Okazaki, T., Bielawska, A., Domae, N., Bell, R. \& Hannun, Y. Characteristics and partial purification of a novel cytosolic, magnesium-independent, neutral sphingomyelinase activated in the early signal transduction of 1 alpha,25-dihydroxyvitamin D3induced HL-60 cell differentiation. J. Biol. Chem. 269, 4070-4077 (1994).

50. Pirianov, G. \& Colston, K. Interactions of vitamin D analogue CB1093, TNFalpha and ceramide on breast cancer cell apoptosis. Mol. Cell Endocrinol. 172, 69-78 (2001).

51. Baudet, C. et al. Differentially expressed genes in C6.9 glioma cells during vitamin D-induced cell death program. Cell Death Differ 5, 116-125 (1998).

52. Baudet, $C$. et al. 1,25-Dihydroxyvitamin D3 induces programmed cell death in a rat glioma cell line. J. Neurosci. Res. 46, 540-550 (1996).

53. Baudet, C. et al. Cytotoxic effects of 1 alpha,25-dihydroxyvitamin D3 and synthetic vitamin D3 analogues on a glioma cell line. Cancer Lett. 100, 3-10 (1996).

54. Maleklou, N., Allameh, A. \& Kazemi, B. Targeted delivery of vitamin D3-loaded nanoparticles to C6 glioma cell line increased resistance to doxorubicin, epirubicin, and docetaxel in vitro. In Vitro Cell Dev. Biol. Anim. 52, 989-1000 (2016).

55. Alvarez-Dolado, M., González-Sancho, J., Navarro-Yubero, C., García-Fernández, L. \& Muñoz, A. Retinoic acid and 1,25-dihydroxyvitamin D3 inhibit tenascin-C expression in rat glioma C6 cells. J. Neurosci. Res. 58, 293-300 (1999).

56. Naveilhan, P. et al. 1,25-Dihydroxyvitamin D3 regulates the expression of the lowaffinity neurotrophin receptor. Brain Res. Mol. Brain Res. 41, 259-268 (1996).

57. Rabizadeh, S. et al. Induction of apoptosis by the low-affinity NGF receptor. Science 261, 345-348 (1993).

58. Schall, T. et al. Molecular cloning and expression of a receptor for human tumor necrosis factor. Cell 61, 361-370 (1990). 
59. Rao, J. Molecular mechanisms of glioma invasiveness: the role of proteases. Nat. Rev. Cancer 3, 489-501 (2003).

60. Suzuki, Y., Fujioka, K., Ikeda, K., Murayama, Y. \& Manome, Y. Temozolomide does not influence the transcription or activity of matrix metalloproteinases 9 and 2 in glioma cell lines. J. Clin. Neurosci. 41, 144-149 (2017).

61. Schiappacassi, M. et al. p27Kip1 expression inhibits glioblastoma growth, invasion, and tumor-induced neoangiogenesis. Mol. Cancer Ther. 7, 1164-1175 (2008).

62. Hu, P. et al. Acidosis enhances the self-renewal and mitochondrial respiration of stem cell-like glioma cells through CYP24A1-mediated reduction of vitamin D. Cell Death Dis. 10, 25 (2019).

63. Naveeilhan, P. et al. Induction of glioma cell death by $1,25(\mathrm{OH}) 2$ vitamin D3: towards an endocrine therapy of brain tumors? J. Neurosci. Res. 37, 271-277 (1994).

64. Friedrich, M. et al. Vitamin D receptor (VDR) expression is not a prognostic factor in cervical cancer. Anticancer Res. 22, 299-304 (2002).

65. Friedrich, M. et al. Vitamin D receptor (VDR) expression is not a prognostic factor in breast cancer. Anticancer Res. 22, 1919-1924 (2002).

66. Villena-Heinsen, C. et al. Immunohistochemical analysis of 1,25-dihydroxyvitaminD3-receptors, estrogen and progesterone receptors and Ki-67 in ovarian carcinoma. Anticancer Res. 22, 2261-2267 (2002).

67. Davoust, N. et al. Vitamin D receptor stable transfection restores the susceptibility to 1,25-dihydroxyvitamin D3 cytotoxicity in a rat glioma resistant clone. J. Neurosci. Res. 52, 210-219 (1998).

68. Neveu, I. et al. 1,25-dihydroxyvitamin D3 regulates the synthesis of nerve growth factor in primary cultures of glial cells. Brain Res. Mol. Brain Res. 24, 70-76 (1994).

69. Neveu, I., Naveilhan, P., Baudet, C., Brachet, P. \& Metsis, M. 1,25-dihydroxyvitamin D3 regulates NT-3, NT-4 but not BDNF mRNA in astrocytes. Neuroreport 6, 124-126 (1994).

70. Naveilhan, P., Neveu, I., Wion, D. \& Brachet, P. 1,25-Dihydroxyvitamin D3, an inducer of glial cell line-derived neurotrophic factor. Neuroreport 7, 2171-2175 (1996).

71. Singer, H., Hansen, B., Martinie, D. \& Karp, C. Mitogenesis in glioblastoma multiforme cell lines: a role for NGF and its TrkA receptors. J. Neurooncol. 45, 1-8 (1999).

72. Brown M., et al. Regulatory effect of nerve growth factor in alpha9beta1 integrindependent progression of glioblastoma. Neuro-oncology.10, 968-980 (2008).

73. Lawn, S. et al. Neurotrophin signaling via TrkB and TrkC receptors promotes the growth of brain tumor-initiating cells. J. Biol. Chem. 290, 3814-3824 (2015).

74. Jaiswal, P., Goel, A. \& Mittal, R. Survivin: a molecular biomarker in cancer. Indian J. Med. Res. 141, 389-397 (2015).

75. Wiesenhofer, B., Weis, C. \& Humpel, C. Glial cell line-derived neurotrophic factor (GDNF) is a proliferation factor for rat C6 glioma cells: evidence from antisense experiments. Antisense Nucleic Acid Drug Dev. 10, 311-321 (2000).

76. Magrassi, L., Butti, G., Pezzotta, S., Infuso, L. \& Milanesi, G. Effects of vitamin D and retinoic acid on human glioblastoma cell lines. Acta Neurochir 133, 184-190 (1995)
77. Lavrenkov, K. National Library of Medicine (US). 201- Aug 13 -. Identifier NCT01181193, High-Dose Vitamin D in Combination With Chemoradiotherapy in the Treatment of Glioblastoma Multiforme (2011). https://clinicaltrials.gov/ct2/ show/study/NCT01181193?term=Vitamin+D\&cond=Glioma\&draw=2\&rank=1.

78. Ramesh K.R. National Library of Medicine (US). 2003 Sep 4 -. Identifier NCT00008086, A Phase I Trial of Subcutaneous And/Or Oral Calcitriol $[(1,25-\mathrm{COH})$ 2D3] and Carboplatin in Advanced Solid Tumors. (2013) https://clinicaltrials.gov/ ct2/show/record/NCT00008086?term=Calcitriol\&cond=Glioma\&draw=2\&rank=1.

79. Tavera-Mendoza, L. et al. Vitamin D receptor regulates autophagy in the normal mammary gland and in luminal breast cancer cells. Proc. Natl. Acad. Sci. USA 114, E2186-E2194 (2017).

80. Toptas, B. et al. The vitamin D receptor (VDR) gene polymorphisms in Turkish brain cancer patients. Biomed. Res. Int. 2013, 295791 (2013).

81. Kure, S. et al. Vitamin D receptor expression is associated with PIK3CA and KRAS mutations in colorectal cancer. Cancer Epidemiol. Biomarkers Prev. 18, 2765-2772 (2009).

\section{AUTHOR CONTRIBUTIONS}

C.L. wrote the paper; K.K. and G.L. reviewed the paper and provided critical revision. All authors read and approved the final paper.

\section{FUNDING INFORMATION}

The authors received no specific funding for this work.

\section{COMPETING INTERESTS}

The authors declare no competing interests.

\section{ETHICS APPROVAL}

This study does not require ethical approval.

\section{ADDITIONAL INFORMATION}

Correspondence and requests for materials should be addressed to Gilberto Ka-Kit Leung.

Reprints and permission information is available at http://www.nature.com/ reprints

Publisher's note Springer Nature remains neutral with regard to jurisdictional claims in published maps and institutional affiliations. 